\title{
Mixed convection heat transfer of water about a vertical surface of variable heat flux with density inversion
}

\author{
RAJENDRA PRASAD SONI and MADHUSUDHANA R GAVARA* \\ Department of Mechanical Engineering, Indian Institute of Technology Guwahati, Guwahati 781039, India \\ e-mail: gmadhu@iitg.ac.in
}

MS received 1 September 2018; revised 15 November 2018; accepted 30 November 2018; published online 14 March 2019

\begin{abstract}
Laminar mixed convection boundary layer flow of water about a vertical plate is studied including density inversion effects. The plate surface is subjected to an arbitrary heat flux that is a function of vertical distance from the leading edge. Both aiding and opposing mixed convection situations are considered in the study. The dimensionless forms of stream function and temperature are expanded in terms of perturbation elements and universal functions. The differentials of the heat flux, which are functions of vertical distance, are used as perturbation elements to obtain universal functions. The results for universal functions required to find temperature and velocity profiles are obtained. The obtained universal functions are valid for any arbitrary wall heat flux variation. The universal applicability of results is demonstrated for power-law variation of wall heat flux. The velocity and temperature variation, boundary layer thickness, Nusselt numbers and skin friction coefficient are presented for various values of mixed convection parameter, wall flux power index, for both aiding and opposing mixed convection. For a given combination of Grashof and Reynolds numbers, the heat transfer rates and skin friction coefficient are found to increase almost linearly with wall flux power index, in the parameter range of the study, for both the cases of aiding and opposing mixed convection. The present results of special cases are found to match well with the results available in the literature.
\end{abstract}

Keywords. Mixed convection; variable wall heat flux; density inversion of water; heat transfer rates.

\section{Introduction}

Density inversion of water has many applications in industries and nature such as refrigeration and atmospheric and oceanic movements. Mixed convection of water near its density inversion plays an important role in refrigeration and air conditioning process, thermal energy storage system using ice for cooling and thermal processing, etc. Water responds to temperature in a peculiar manner near its density maximum in a certain temperature range near $4^{\circ} \mathrm{C}$. Density of water increases with increasing temperature near its density maximum, which is unlike the usual density variation of fluids, which decreases with temperature. This increasing density with temperature is called density inversion. Water density varies non-linearly in this temperature range, unlike the usual linear variation outside this temperature range. This peculiar density variation introduces complex behaviour in the convective characteristics. Hence, the study of effect of density inversion is important, which is the aim of the present study.

Mixed convection boundary layer flow over vertical wall with uniform heat flux surface condition was numerically studied in [1-3]. Wilks [1] presented series solutions for

*For correspondence leading and trailing edges, which are valid for Prandtl number of order 1 . For the same mixed convection problem, Carey and Gebhart [2] considered higher-order series solutions. They compared their results with those of Wilks [1] at trailing edge and found that Wilks results were of lower accuracy due to first-order approximation. An accurate finite-difference marching technique was used to investigate the effect of buoyancy in forced convection flow over vertical wall by Moulic and Yao [3]. It was found that forced convection was the dominant mode of heat transfer at the leading edge, whereas free convection prevailed at the trailing edge of the plate. Merkin and Mahmood [4] used similarity solutions to analyse the effect of buoyancy parameter on mixed convection flows. It was reported that solutions approach free convection asymptote for large values of buoyancy parameter in aiding flows, while dual solutions exist for opposing flows. Consequently, the same problem was extended in Merkin et al [5] to study the Prandtl number effect on mixed convection. The results imply that, for fixed values of other parameters, solutions approach pure forced convection with increasing Prandtl number.

The orientation of the surface shows strong influence on mixed convection. Such an effect was the subject of several studies. The effect of plate orientation on mixed convection 
flows was numerically studied by Wickern [6] for both aiding and opposing flows. Flow separations were reported for opposing flow situation, which could be singular or regular. The same author extended the study, in [7], to investigate the effect of Prandtl number on mixed convection. The convection characteristics were studied using an asymptotic expansion for wide range of Prandtl number and compared to results obtained using a numerical method. The asymptotic analysis results were consistent with the numerical results. Yeh et al [8] analysed the effect of Prandtl number, buoyancy-induced pressure gradient and ratio of wall to free-stream velocity on mixed convection over a horizontal plate using a local non-similarity method. The results showed profound influence of buoyancy on overall heat transfer for certain ranges of parameters. Armaly et al [9] analytically studied the combined effect of free and forced convection over heated vertical surface for a wide range of Prandtl number. They presented the correlations for local and average Nusselt number for both aiding and opposing flow situations. Mixed convection flow over a vertical plate with specified heat flux in water with nanofluid was studied by Trimbitas et al [10] using a similarity solution. Higher values of skin friction coefficient and heat transfer rates were found with inclusion of nanofluids in water, compared with those without nanofluids.

Mixed convection in porous medium was the focus of many studies. A finite-difference scheme was used by Ranganathan and Viskanta [11] to study the mixed convection boundary layer over a vertical surface in a porous medium. Various parameters such as Prandtl number, $\mathrm{Gr} / \mathrm{Re}$ and low blowing velocity showed significant impact on velocity and temperature distributions, while porosity variation showed insignificant effect. Mixed convection boundary layer flow in a porous medium over vertical plate was numerically investigated by Ahmad and Pop [12] for various nanofluids. For the same parameter values, significant amount of heat transfer enhancement was registered when nanofluids were used compared with that without nanofluids.

In many works, the radiation effect is included in the mixed convection study. The effects of variable viscosity and thermal conductivity of air along with the effect of radiation over a non-isothermal horizontal permeable surface on fluid flow and heat transfer were studied by Mahmoud [13]. The local skin friction coefficient and Nusselt number were found to increase with increasing values of radiation and suction parameters, while decreasing with increasing value of blowing parameter. Combined effect of conduction, mixed convection and surface radiation from a vertical plate with internal heat generation was numerically studied by Sawant and Rao [14]. The effects of surface emissivity and modified Richardson number on fluid flow behaviours were investigated. Correlations were presented for average temperature, average Nusselt number and skin friction coefficients for wide ranges of parameters. The effect of thermal radiation and variable heat source or sink on unsteady mixed convection on stretching permeable sheet was studied by Pal [15]. The effect of Prandtl number, buoyancy, radiation and unsteadiness parameters on aiding and opposing mixed convection was studied. The local Nusselt number increased with increasing values of unsteadiness parameter, buoyancy parameter and Prandtl number.

Several authors studied the effect of density inversion of water on the convection characteristics of water flows over solid surfaces. Vighnesam and Soundalgekar [16] studied mixed convection flow of water over a vertical surface of variable temperature near density inversion. The authors presented similarity solutions for velocity, temperature and Nusselt number variations for various values of modified Richardson number. Numerical study of free convection flows over an isothermal horizontal plate in a porous medium filled with water was carried out by Lin and Gebhart [17]. The authors studied the effect of density inversion parameter on free convection and reported the existence of reversal of normal component of velocity in the inner region of flow. Kumaran and Pop [18] investigated free convection boundary layer flows of water near its density inversion over a vertical plate in a porous medium for various wall thermal conditions such as variable wall temperature, variable heat flux conditions, etc., and presented similarity solutions for the flow and thermal field.

Many studies in the literature examined mixed convection boundary layer for fluids without density inversion. The effect of density inversion in mixed convection flows of water has received less attention in the literature. Hence, in the present study, mixed convection boundary layer of water about a vertical flat plate with arbitrarily varying surface heat flux is studied using perturbation method. Various other methods such as power series method, similarity method and numerical method are used in different studies in the literature such as $[2,4,19,20]$ for solving boundary layer flows. The perturbation method is also applied to boundary layer convective flows in several studies. Recently, Gavara et al [21] applied perturbation method to the boundary layer mixed convection flow over a non-isothermal vertical flat plate. Seetharamu and Dutta [22] solved boundary layer mixed convection in a porous medium about a vertical wall with non-uniform temperature. The perturbation method has the inherent advantage that the solutions obtained from the method are valid for any type of wall temperature or wall heat flux variation. Hence, perturbation technique is chosen in the present study to solve the boundary layer mixed convection flows.

\section{Physical system}

The physical system consists of a vertical flat plate. Water flows over the plate such that mixed convection boundary layer is formed. The surface of the plate is subjected to wall heat flux varying as an arbitrary function of vertical distance. The free stream velocity and temperature are $v_{\infty}$ and $T_{0}$, respectively, where $T_{0}$ is the temperature corresponding 
to the density maximum point near density inversion. The temperatures of the plate and water are in the range of temperatures in which water shows density inversion. Schematics of the physical and coordinate systems are shown in figure $1 \mathrm{a}$ and $\mathrm{b}$ for aiding and opposing mixed convection conditions, respectively.

\section{Mathematical model}

In the present study, mixed convection is assumed to be steady, laminar and incompressible. The flow is assumed to be such that boundary layer approximations are valid. The viscous dissipation and radiation effects are assumed to be negligible. The properties of the water are considered to be constant except for the density variation with temperature in buoyancy term. The temperatures within the boundary layer are assumed to be in the range of temperatures in which water shows density inversion. The density variation of water with temperature in the buoyancy term is modelled with different functions in different studies such as $[16,18,23]$. In the present study, the density variation considered in the work of [23] is adopted, which is given by

$$
\frac{\rho}{\rho_{0}}=1-\gamma\left(T-T_{0}\right)^{2}
$$

where $\rho_{0}$ is the maximum density at the temperature of $T_{0}=3.98^{\circ} \mathrm{C}$ and $\gamma=8.0 \times 10^{-6 \circ} \mathrm{C}^{-2}$ is a constant. This correlation is within acceptable error limit in temperature range $0-12^{\circ} \mathrm{C}$ [23]. With the above considerations, governing equations for boundary layer flows take the following form:

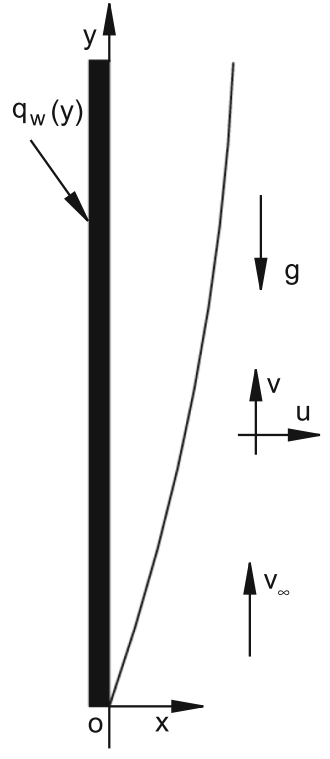

(a) Aiding mixed convection

Figure 1. Schematic of physical and coordinate systems. continuity equation

$$
\frac{\partial u}{\partial x}+\frac{\partial v}{\partial y}=0
$$

$y$-momentum equation

$$
u \frac{\partial v}{\partial x}+v \frac{\partial v}{\partial y}=v \frac{\partial^{2} v}{\partial x^{2}} \pm g \gamma\left(T-T_{0}\right)^{2}
$$

energy equation

$$
u \frac{\partial T}{\partial x}+v \frac{\partial T}{\partial y}=\alpha \frac{\partial^{2} T}{\partial x^{2}}
$$

where $\alpha$ is thermal diffusivity of water. The ' + ' and '-' signs associated with body force term in Eq. (3) represent the aiding and opposing mixed convection flows, respectively. These governing equations are subjected to following boundary conditions. No slip and no penetration conditions prevail at the plate surface. The velocity and temperature outside the boundary layer are uniform at $v_{\infty}$ and $T_{0}$, respectively. These boundary conditions can be mathematically expressed as follows.

$$
\begin{aligned}
& \text { At } \quad \mathrm{x}=0: \quad \mathrm{u}=\mathrm{v}=0, \quad \mathrm{q}=\mathrm{q}_{\mathrm{w}}(\mathrm{y}) . \\
& \text { At } \quad \mathrm{x} \rightarrow \infty: \quad \mathrm{v} \rightarrow \mathrm{v}_{\infty}, \quad \mathrm{T} \rightarrow \mathrm{T}_{0} .
\end{aligned}
$$

To convert these governing equations in the form of stream function, the velocity components are expressed in stream function as

$$
u=-\frac{\partial \psi}{\partial y}, \quad v=\frac{\partial \psi}{\partial x} .
$$

Substituting Eq. (7) in Eqs. (3) and (4), the resulting stream function forms of momentum and energy equations are

$$
\begin{gathered}
\frac{\partial \psi}{\partial x} \frac{\partial^{2} \psi}{\partial x \partial y}-\frac{\partial \psi}{\partial y} \frac{\partial^{2} \psi}{\partial x^{2}}=v \frac{\partial^{3} \psi}{\partial x^{3}} \pm g \gamma\left(T-T_{0}\right)^{2}, \\
\frac{\partial \psi}{\partial x} \frac{\partial T}{\partial y}-\frac{\partial \psi}{\partial y} \frac{\partial T}{\partial x}=\alpha \frac{\partial^{2} T}{\partial x^{2}} .
\end{gathered}
$$

The boundary conditions in stream function form take the following form.

$$
\begin{aligned}
& \text { At } \quad \mathrm{x}=0: \quad \frac{\partial \psi}{\partial \mathrm{x}}=\frac{\partial \psi}{\partial \mathrm{y}}=0, \quad \mathrm{q}=\mathrm{q}_{\mathrm{w}}(\mathrm{y}) \\
& \text { At } \quad \mathrm{x} \rightarrow \infty: \quad \frac{\partial \psi}{\partial \mathrm{x}} \rightarrow \mathrm{v}_{\infty}, \quad \mathrm{T} \rightarrow \mathrm{T}_{0}
\end{aligned}
$$

\section{Solutions of the equations}

To seek perturbation type of solutions, the governing equations (8) and (9) need to be suitably transformed. The momentum and energy equations are transformed using the following dimensionless variables: 


$$
\begin{gathered}
f\left(\eta, \lambda_{0}, \lambda_{1}, \cdots, \lambda_{n}, \cdots\right)=\frac{\psi(x, y)}{\sqrt{v v_{\infty} y}}, \\
\theta\left(\eta, \lambda_{0}, \lambda_{1}, \cdots, \lambda_{n}, \cdots\right)=\frac{T(x, y)-T_{0}}{\frac{q_{w} y}{k} \sqrt{\frac{v}{v_{\infty} y}}}, \\
\eta(x, y)=x \sqrt{\frac{v_{\infty}}{v y}} .
\end{gathered}
$$

Here, $\lambda_{0}, \lambda_{1}, \ldots, \lambda_{n}$ are a set of infinite number of variables that are functions of $y$. These variables are the perturbation elements, which are yet unknown functions of $y$. These transformations closely resemble the similarity transformations except that in the present form, additional set of functions $\lambda_{n}(y)$ are present. Applying these transformations, the governing equations become

$$
\begin{aligned}
\frac{\partial^{3} f}{\partial \eta^{3}}= & -\frac{f}{2} \frac{\partial^{2} f}{\partial \eta^{2}} \pm\left(\frac{G r_{y}}{R e_{y}^{3}}\right) \theta^{2}+\frac{\partial^{2} f}{\partial \eta^{2}} y \sum_{n=0}^{\infty} \frac{\partial f}{\partial \lambda_{n}} \frac{\partial \lambda_{n}}{\partial y} \\
& -\frac{\partial f}{\partial \eta} y \sum_{n=0}^{\infty} \frac{\partial^{2} f}{\partial \lambda_{n} \partial \eta} \frac{\partial \lambda_{n}}{\partial y} \\
\frac{1}{\operatorname{Pr}} \frac{\partial^{2} \theta}{\partial \eta^{2}}= & -\frac{f}{2} \frac{\partial \theta}{\partial \eta}+\frac{\partial f}{\partial \eta} y \sum_{n=0}^{\infty} \frac{\partial \theta}{\partial \lambda_{n}} \frac{\partial \lambda_{n}}{\partial y} \\
& -\frac{\partial \theta}{\partial \eta} y \sum_{n=0}^{\infty} \frac{\partial f}{\partial \lambda_{n}} \frac{\partial \lambda_{n}}{\partial y}+y \frac{\partial f}{\partial \eta} \frac{\theta}{q_{w}(y)} \frac{d}{d y} q_{w}(y)
\end{aligned}
$$

where the modified Grashof number is given by

$$
G r_{y}=\frac{g \gamma\left(q_{w} y / k\right)^{2} y^{3}}{v^{2}}
$$

and Reynolds number is given by

$$
R e_{y}=\frac{v_{\infty} y}{v}
$$

The difference in this form of expression for modified Grashof number compared with the usual form of Grashof number is due to the presence of density inversion effect, being represented by $\gamma$ and given by Eq. (1), which brings peculiarities.

From these equations, we can see that $G r_{y} / R e_{y}^{3}$ is an important governing parameter of the mixed convection with density inversion. The boundary conditions in terms of dimensionless variables $f$ and $\theta$ are as follows.

$$
\begin{gathered}
\text { At } \quad \eta=0: \quad \mathrm{f}=0, \quad \frac{\partial \mathrm{f}}{\partial \eta}=0, \quad \frac{\partial \theta}{\partial \eta}=-1 . \\
\text { At } \quad \eta \rightarrow \infty: \quad \frac{\partial \mathrm{f}}{\partial \eta} \rightarrow 1, \quad \theta \rightarrow 0 .
\end{gathered}
$$

Study of Eqs. (15) and (16) reveals that the equations become functions of $\eta$ and $\lambda_{n}$, if we define $\lambda_{n}(y)$ as [22]

$$
\lambda_{n}=\frac{y^{n+1}}{q_{w}(y)} \frac{d^{n+1}}{d y^{n+1}}\left(q_{w}(y)\right) .
$$

Here, $q_{w}(y)$ is infinitely differentiable with respect to $y$. After substitution of $\lambda_{n}$ from Eq. (21), the governing equations (15) and (16) are still in the form of partial differential equations. To convert the partial differential equations into ordinary differential equations, the functions $f$ and $\theta$ are expressed in following power series of $\lambda_{n}(y)$ with coefficients that are functions of $\eta$ :

$$
\begin{aligned}
& f\left(\eta, \lambda_{0}, \lambda_{1}, \ldots, \lambda_{n}, \ldots\right)=F(\eta)+\lambda_{0} f_{0}(\eta) \\
& \quad+\lambda_{1} f_{1}(\eta)+\cdots+\lambda_{0}^{2} f_{00}(\eta)+\lambda_{1}^{2} f_{11}(\eta)+\cdots, \\
& \theta\left(\eta, \lambda_{0}, \lambda_{1}, \cdots, \lambda_{n}, \cdots\right)=H(\eta)+\lambda_{0} \theta_{0}(\eta) \\
& \quad+\lambda_{1} \theta_{1}(\eta)+\cdots+\lambda_{0}^{2} \theta_{00}(\eta)+\lambda_{1}^{2} \theta_{11}(\eta)+\cdots
\end{aligned}
$$

Substituting Eqs. (21)-(23) in Eqs. (15) and (16), we get the governing equations in the following form:

$$
\begin{aligned}
F^{\prime \prime \prime} & +\lambda_{0} f_{0}^{\prime \prime \prime}+\lambda_{1} f_{1}^{\prime \prime \prime}+\cdots+\lambda_{0}^{2} f_{00}^{\prime \prime \prime}+\cdots=-\frac{1}{2} F F^{\prime \prime} \\
& +\frac{G r_{y}}{R e_{y}^{3}} H^{2}+\lambda_{0}\left(-\frac{1}{2} F f_{0}^{\prime \prime}-\frac{3}{2} f_{0} F^{\prime \prime} \pm \frac{G r_{y}}{R e_{y}^{3}} 2 H \theta_{0}\right. \\
& \left.+F^{\prime} f_{0}^{\prime}\right)+\lambda_{1}\left(-\frac{1}{2} F f_{1}^{\prime \prime}-\frac{5}{2} f_{1} F^{\prime \prime}-F^{\prime \prime} f_{0}+F^{\prime} f_{0}\right. \\
& \left.+\frac{G r_{y}}{R e_{y}^{3}} 2 H \theta_{1}+2 F^{\prime} f_{1}^{\prime}\right)+\lambda_{0}^{2}\left(-\frac{1}{2} F f_{00}^{\prime \prime}-\frac{5}{2} f_{00} F^{\prime \prime}\right. \\
& -\frac{3}{2} f_{0} f_{0}^{\prime \prime} \pm \frac{G r_{y}}{R e_{y}^{3}}\left(H \theta_{00}+2 H \theta_{0}\right)+F^{\prime \prime} f_{0} \\
+ & \left.2 F^{\prime} F_{00}^{\prime}+f_{0}^{\prime} f_{0}^{\prime}-F^{\prime} f_{0}^{\prime}\right), \\
\frac{1}{P r} & \left(H^{\prime \prime}+\lambda_{0} \theta_{0}^{\prime \prime}+\lambda_{1} \theta_{1}^{\prime \prime}+\cdots+\lambda_{0}^{2} \theta_{00}^{\prime \prime}+\cdots\right)=-\frac{1}{2} F H^{\prime} \\
& +\frac{1}{2} F^{\prime} H+\lambda_{0}\left(-\frac{1}{2} F \theta_{0}^{\prime}+F^{\prime} \theta_{0}-\frac{3}{2} f_{0} H^{\prime}\right. \\
& \left.+H F^{\prime}+\frac{1}{2} f_{0} H^{\prime}+\frac{1}{2} F^{\prime} \theta_{0}\right)+\lambda_{1}\left(-\frac{1}{2} F \theta_{1}^{\prime}\right. \\
& \left.+\frac{1}{2} \theta_{0} f_{0}^{\prime}+\frac{1}{2} F^{\prime} \theta_{00}\right) \\
& -\frac{5}{2} f_{1} H^{\prime}+F^{\prime} \theta_{0}+2 F^{\prime} \theta_{1}-H^{\prime} f_{0}+\frac{1}{2} F^{\prime} \theta_{1} \\
& \left.+\frac{1}{2} f_{1}^{\prime} H\right)+\lambda_{0}^{2}\left(-\frac{1}{2} F \theta_{00}^{\prime}-\frac{5}{2} f_{00} H^{\prime}+2 F^{\prime} \theta_{00}\right. \\
& +H^{\prime} f_{0}+H f_{0}+\frac{1}{2} f_{00}^{\prime} H
\end{aligned}
$$

In Eqs. (24) and (25), if we equate the coefficient of $\lambda_{0}$ on either sides of the governing equations, we obtain a set of ordinary differential equations of coefficient functions. Similarly, equating the coefficients of $\lambda_{1}, \lambda_{0}^{2}, \ldots$, other sets of 
ordinary differential equations will be obtained. Similarly, an infinite number of sets of ordinary differential equations can be obtained by equating the coefficients of same power of $\lambda_{n}(y)$ variables. These sets of equations are coupled equations. In the calculations, larger the number of equation sets considered, higher the accuracy of results we obtain [24, 25]. It is found in the calculation that when we include beyond sets of equations corresponding to constant $\lambda_{0}, \lambda_{1}$ and $\lambda_{0}^{2}$, the change in the solution is found to be negligible. Hence, the sets correspond to constant, $\lambda_{0}, \lambda_{1}$ and $\lambda_{0}^{2}$ only are considered in the present study, which are as follows.

Constant terms:

$$
\begin{aligned}
F^{\prime \prime \prime} & =-\frac{1}{2} F F^{\prime \prime} \pm\left(\frac{G r}{R e^{3}}\right) H^{2} \\
\frac{1}{P r} H^{\prime \prime} & =-\frac{1}{2} F H^{\prime}+\frac{1}{2} F^{\prime} H .
\end{aligned}
$$

The set of coefficients of $\lambda_{0}$ :

$$
\begin{aligned}
f_{0}^{\prime \prime \prime}= & -\frac{1}{2} F f_{0}^{\prime \prime}-\frac{3}{2} f_{0} F^{\prime \prime} \\
& \pm\left(\frac{G r}{R e^{3}}\right) 2 H \theta_{0}+F^{\prime} f_{0}^{\prime} \\
\frac{1}{P r} \theta_{0}^{\prime \prime}= & -\frac{1}{2} F \theta_{0}^{\prime}-\frac{3}{2} f_{0} H^{\prime}+F^{\prime} \theta_{0}+H F^{\prime} \\
& +\frac{1}{2} f_{0} H^{\prime}+\frac{1}{2} F^{\prime} \theta_{0} .
\end{aligned}
$$

The set of coefficient of $\lambda_{1}$ :

$$
\begin{aligned}
f_{1}^{\prime \prime \prime}= & -\frac{1}{2} F f_{1}^{\prime \prime}-\frac{5}{2} f_{1} F^{\prime \prime} \pm\left(\frac{G r}{R e^{3}}\right) 2 H \theta_{1} \\
& -F^{\prime \prime} f_{0}+F^{\prime} f_{0}^{\prime}+2 F^{\prime} f_{1}^{\prime} \\
\frac{1}{P r} \theta_{1}^{\prime \prime}= & -\frac{1}{2} F \theta_{1}^{\prime}-\frac{5}{2} f_{1} H^{\prime}+F^{\prime} \theta_{0}+2 F^{\prime} \theta_{1} \\
& -H^{\prime} f_{0}+\frac{1}{2} F^{\prime} \theta_{1}+\frac{1}{2} f_{1}^{\prime} H .
\end{aligned}
$$

The set of coefficient of $\lambda_{0}^{2}$ :

$$
\begin{aligned}
f_{00}^{\prime \prime \prime}= & -\frac{1}{2} F f_{00}^{\prime \prime}-\frac{5}{2} f_{00} F^{\prime \prime}-\frac{3}{2} f_{0} f_{0}^{\prime \prime} \\
& \pm\left(\frac{G r}{R e^{3}}\right)\left(\theta_{0}^{2}+2 H \theta_{00}\right)+F^{\prime \prime} f_{0} \\
& +2 F^{\prime} F_{00}^{\prime}+f_{0}^{\prime} f_{0}^{\prime}-F^{\prime} f_{0}^{\prime} \\
\frac{1}{P r} \theta_{00}^{\prime \prime}= & -\frac{1}{2} F \theta_{00}^{\prime}-\frac{5}{2} f_{00} H^{\prime}+2 F^{\prime} \theta_{00} \\
& -\frac{3}{2} f_{0} \theta_{0}^{\prime}+f_{0}^{\prime} \theta_{0}+H^{\prime} f_{0}+H f_{0}^{\prime} \\
& +\frac{1}{2} f_{00}^{\prime} H+\frac{1}{2} \theta_{0} f_{0}^{\prime}+\frac{1}{2} F^{\prime} \theta_{00} .
\end{aligned}
$$

The sets of differential equations corresponding to other $\lambda_{n}$ and higher powers of $\lambda_{n}$ contribute negligibly small to the solutions, and hence they are neglected. The '+' and '-' signs associated with $G r_{y} / R e_{y}^{3}$ in these equations correspond to aiding and opposing mixed convection flows, respectively. The boundary conditions for these differential equations are as follows.

$$
\begin{aligned}
& \text { At } \quad \eta=0: \quad F=0, \quad f_{0}=f_{1}=f_{00}=f_{2}=0, \\
& H^{\prime}=-1, \quad \theta_{0}^{\prime}=\theta_{1}^{\prime}=\theta_{00}^{\prime}=\theta_{2}^{\prime}=0 \text {, } \\
& F^{\prime}=0, \quad f_{0}^{\prime}=f_{1}^{\prime}=f_{00}^{\prime}=f_{2}^{\prime}=0 \text {. } \\
& \text { At } \quad \eta \rightarrow \infty: \quad \begin{array}{l}
F^{\prime}=1, \quad f_{0}^{\prime}=f_{1}^{\prime}=f_{00}^{\prime}=f_{2}^{\prime}=0, \\
H=0, \quad \theta_{0}=\theta_{1}=\theta_{00}=\theta_{2}=0 .
\end{array}
\end{aligned}
$$

The afore-mentioned sets of coupled equations with these boundary conditions are solved using the shooting method to find the coefficient functions. A fourth-order Runge-Kutta method is used for the integration of ordinary differential equations. The value of Prandtl number of water near density inversion is taken as $11.4 \mathrm{in}$ the calculations. The solutions of these equations yield the values of coefficient functions for various $\eta$ values. These coefficient functions are obtained for any arbitrary wall heat flux variation and hence, they are valid for any continuous wall heat flux variation. They are independent of type of wall heat flux variation and hence, they are called "universal functions" for a given fluid. The obtained universal functions for stream function and temperature are shown in figures 2, 3 and 4 for various values of $G r_{y} / R e_{y}^{3}$. Figure 2 for $G r_{y} / R e_{y}^{3}=0$ corresponds to forced convection asymptote. Figures 3 and 4 show the universal functions of stream function and temperature, respectively, for aiding and opposing mixed convection flows.

\section{Velocity, skin friction coefficient and Nusselt number in terms of universal functions}

In mixed convection flows, the objective is to find important quantities, which are velocity and temperature distribution in the boundary layer, and drag and heat transfer rates along the wall. The temperature distribution is given by Eq. (23). The velocity, drag in the form of skin friction coefficient and heat transfer rates in the form of Nusselt number can be expressed in terms of universal functions. The relations can be obtained as follows.

The vertical component of the velocity can be expressed as $v=\frac{\partial \psi}{\partial x}$. Using Eqs. (12) and (14) in this expression, $v$ becomes

$$
v=\frac{\partial \psi}{\partial x}=\sqrt{v_{\infty} v y} \frac{\partial f}{\partial \eta} \frac{\partial \eta}{\partial x}=v_{\infty} f^{\prime}(\eta) .
$$


Using Eq. (22) in this equation, vertical velocity in terms of universal functions can be expressed as

$$
\begin{aligned}
\frac{v}{v_{\infty}}=f^{\prime}(\eta)= & F^{\prime}(\eta)+\lambda_{0} f_{0}^{\prime}(\eta)+\lambda_{1} f_{1}^{\prime}(\eta)+\cdots \\
& +\lambda_{0}^{2} f_{00}^{\prime}(\eta)+\cdots
\end{aligned}
$$

To calculate skin friction drag, the local skin friction coefficient can be expressed as

$$
C_{f y}=\frac{\tau_{\mathrm{w}}}{\rho v_{\infty}^{2}}
$$

where $v_{\infty}$ is free-stream velocity and $\tau_{\mathrm{w}}$ is shear stress at wall $(x=0)$. The wall shear stress can be expressed as

$$
\tau_{\mathrm{w}}=\left.\mu \frac{\partial v}{\partial x}\right|_{x=0}=\left.\mu \frac{\partial}{\partial x}\left(\frac{\partial \psi}{\partial x}\right)\right|_{x=0}=\left.\mu v_{\infty} \sqrt{\frac{v_{\infty}}{v y \partial \eta^{2}}}\right|_{\eta=0} .
$$

Using Eqs. (34) and (35) we get

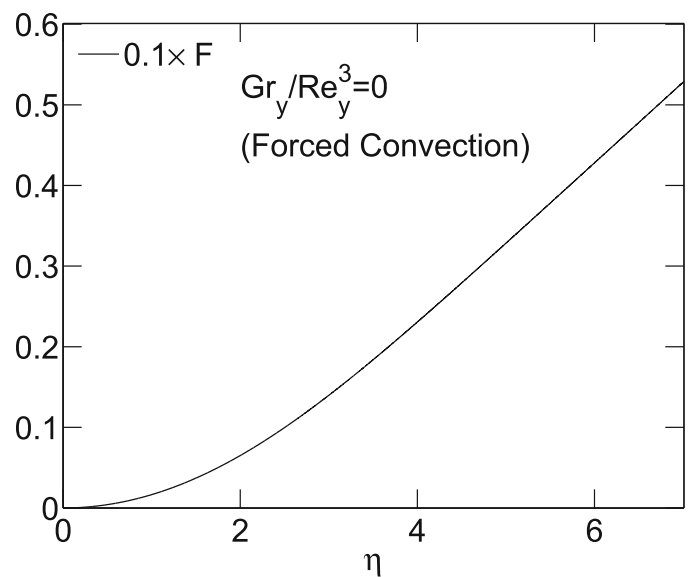

(a) $G r_{y} / R e_{y}^{3}=0$

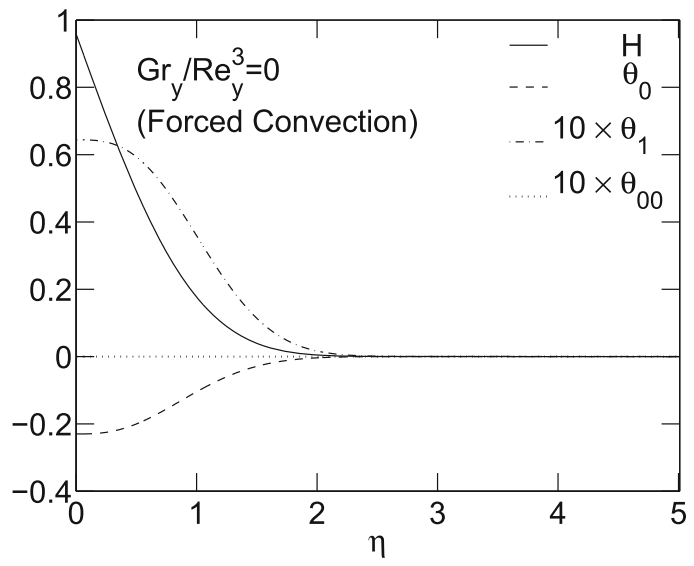

(b) $G r_{y} / R e_{y}^{3}=0$

Figure 2. Variation of universal functions of stream function and temperature for forced convection asymptote.

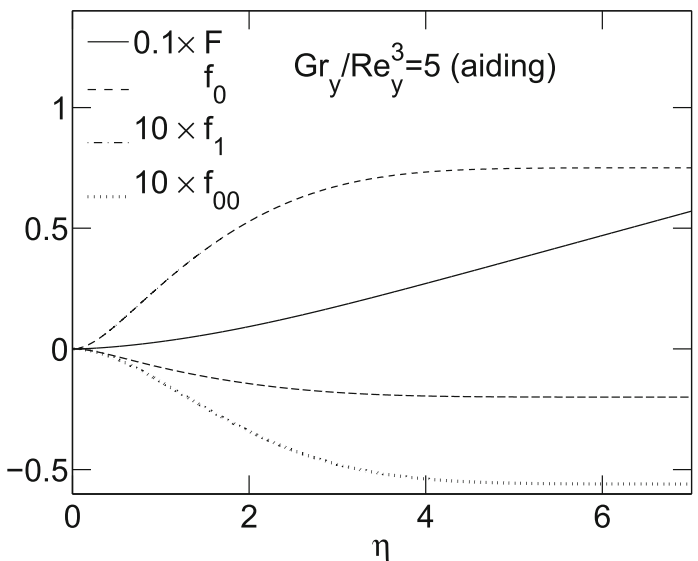

(a) $G r_{y} / R e_{y}^{3}=5$

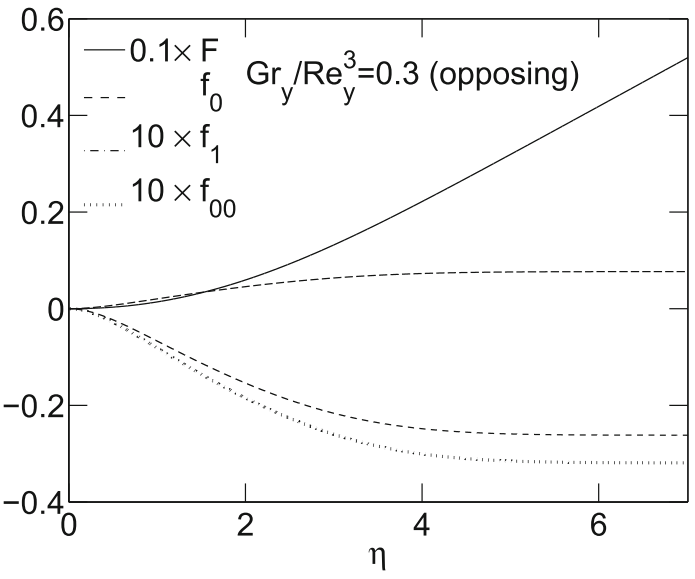

(b) $G r_{y} / R e_{y}^{3}=0.3$

Figure 3. Universal functions of stream function for $G r_{y} / R e_{y}^{3}$ values in aiding and opposing flows.

$$
\begin{aligned}
\frac{C_{f y}}{\operatorname{Re}_{y}^{-1 / 2}}= & \left.f^{\prime \prime}(\eta)\right|_{\eta=0}=F^{\prime \prime}(0)+\lambda_{0} f_{0}^{\prime \prime}(0) \\
& +\lambda_{1} f_{1}^{\prime \prime}(0)+\cdots+\lambda_{0}^{2} f_{00}^{\prime \prime}(0)+\cdots
\end{aligned}
$$

Similarly, the relation between local heat transfer coefficient $h(y)$ and local wall heat flux is given as

$$
h(y)=\frac{q_{w}(y)}{T_{w}-T_{0}} .
$$

Heat transfer rates are expressed in terms of local Nusselt number, which is expressed as

$$
N u_{y}=\frac{h(y) y}{k} .
$$

Combining Eqs. (37) and (38), we get

$$
N u_{y}=\frac{q_{w}(y)}{T_{w}-T_{0}} \frac{y}{k} .
$$




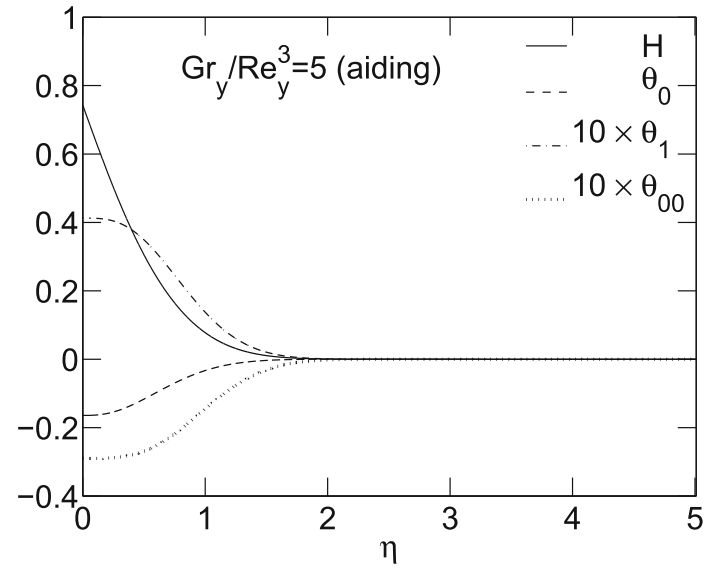

(a) $G r_{y} / R e_{y}^{3}=5$

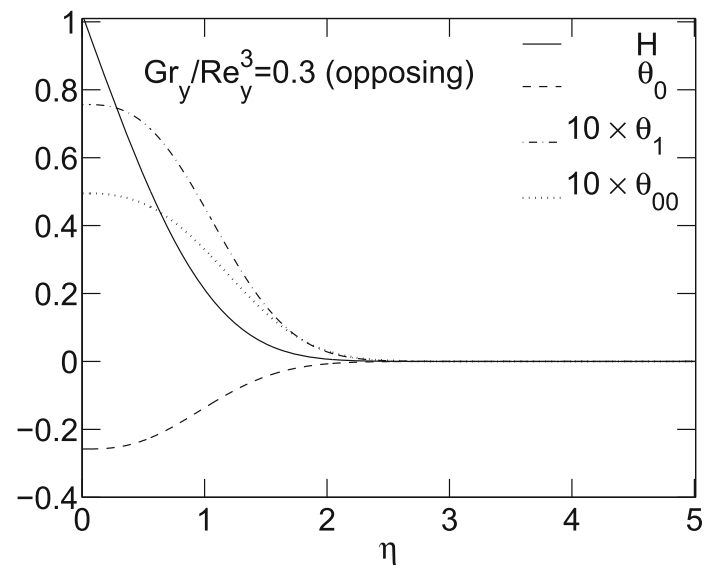

(b) $G r_{y} / R e_{y}^{3}=0.3$

Figure 4. Universal functions of temperature for $G r_{y} / R e_{y}^{3}$ values in aiding and opposing flows.

From the definition of dimensionless temperature given by Eq. (13) we can express $\left(T_{w}-T_{0}\right)$ as

$$
T_{w}-T_{0}=\theta_{\eta=0} \frac{q_{w}(y)}{k} y R e_{y}^{-1 / 2}
$$

where $\theta_{\eta=0}$ is the dimensionless temperature corresponding to wall, for which $x=0$ and hence $\eta=0$. Substituting Eq. (40) in Eq. (39), we get the heat transfer rate as

$$
\frac{N u_{y}}{R e_{y}^{1 / 2}}=\frac{1}{\theta_{\eta=0}} .
$$

The heat transfer rates in Eq. (41) can be expressed in the form of universal constants, using Eq. (23), as

$$
\begin{aligned}
\frac{N u_{y}}{R e_{y}^{1 / 2}}= & {\left[H(0)+\lambda_{0} \theta_{0}(0)+\lambda_{1} \theta_{1}(0)+\cdots\right.} \\
& \left.+\lambda_{0}^{2} \theta_{00}(0)+\cdots\right]^{-1}
\end{aligned}
$$

Table 1. Values of universal constants of stream function for various $G r_{y} / R e_{y}^{3}$ values (aiding flow).

\begin{tabular}{lcccc}
\hline$G r_{y} / R e_{y}^{3}$ & $F^{\prime \prime}(0)$ & \multicolumn{1}{c}{$f_{0}^{\prime \prime}(0)$} & $f_{1}^{\prime \prime}(0)$ & \multicolumn{1}{c}{$f_{00}^{\prime \prime}(0)$} \\
\hline 0 & 0.3321 & 0.0000 & 0.0000 & 0.0000 \\
0.05 & 0.3477 & -0.0100 & 0.0031 & 0.0013 \\
0.5 & 0.4658 & -0.0815 & 0.0247 & 0.0030 \\
1 & 0.5694 & -0.1406 & 0.0423 & -0.0014 \\
5 & 0.9865 & -0.3632 & 0.1073 & -0.0334 \\
10 & 1.5105 & -0.6302 & 0.1842 & -0.0804 \\
20 & 2.1153 & -0.9327 & 0.2708 & -0.1366 \\
\hline
\end{tabular}

Table 2. Values of universal constants of stream function for various $G r_{y} / R e_{y}^{3}$ values (opposing flow).

\begin{tabular}{lccrr}
\hline$G r_{y} / R e_{y}^{3}$ & $F^{\prime \prime}(0)$ & $f_{0}^{\prime \prime}(0)$ & \multicolumn{1}{c}{$f_{1}^{\prime \prime}(0)$} & \multicolumn{1}{c}{$f_{00}^{\prime \prime}(0)$} \\
\hline 0 & 0.3321 & 0.0000 & 0.0000 & 0.0000 \\
0.05 & 0.3157 & 0.0106 & -0.0033 & -0.0018 \\
0.1 & 0.2984 & 0.0220 & -0.0068 & -0.0042 \\
0.2 & 0.2605 & 0.0480 & -0.0149 & -0.0125 \\
0.3 & 0.2162 & 0.0802 & -0.0251 & -0.0300 \\
\hline
\end{tabular}

Table 3. Values of universal constants of temperature for aiding mixed convection flow for various $G r_{y} / R e_{y}^{3}$ values.

\begin{tabular}{lcccr}
\hline$G r_{y} / R e_{y}^{3}$ & $H(0)$ & $\theta_{0}(0)$ & $\theta_{1}(0)$ & $\theta_{00}(0)$ \\
\hline 0 & 0.9591 & -0.2301 & 0.0644 & 0.0000 \\
0.05 & 0.9516 & -0.2272 & 0.0633 & -0.0032 \\
0.5 & 0.9017 & -0.2095 & 0.0566 & -0.0178 \\
1 & 0.8656 & -0.1980 & 0.0525 & -0.0234 \\
5 & 0.7436 & -0.1644 & 0.0412 & -0.0290 \\
10 & 0.6826 & -0.1494 & 0.0368 & -0.0286 \\
20 & 0.6214 & -0.1352 & 0.0327 & -0.0273 \\
\hline
\end{tabular}

Equations (36) and (42) show that $C_{f y} / R e_{y}^{-1 / 2}$ and $N u_{y} / R e_{y}^{1 / 2}$ depend on universal functions of stream function $\left(F^{\prime \prime}(0), f_{0}^{\prime \prime}(0), f_{1}^{\prime \prime}(0), f_{00}^{\prime \prime}(0), \ldots\right)$ and temperature $(H(0)$, $\left.\theta_{0}(0), \theta_{1}(0), \theta_{00}(0), \ldots\right)$, respectively, as well as the perturbation variables $\lambda_{0}, \lambda_{1}, \lambda_{0}^{2}, \ldots$. Since universal constants are the same for all boundary conditions, the skin friction drag and heat transfer rates depend on the perturbation

Table 4. Values of universal constants of temperature for opposing mixed convection flow for various $G r_{y} / R e_{y}^{3}$ values.

\begin{tabular}{lcccc}
\hline$G r_{y} / R e_{y}^{3}$ & $H(0)$ & $\theta_{0}(0)$ & $\theta_{1}(0)$ & $\theta_{00}(0)$ \\
\hline 0 & 0.9591 & -0.2301 & 0.0644 & 0.0000 \\
0.05 & 0.9673 & -0.2332 & 0.0656 & 0.0039 \\
0.1 & 0.9761 & -0.2368 & 0.0671 & 0.0088 \\
0.2 & 0.9968 & -0.2456 & 0.0706 & 0.0230 \\
0.3 & 1.0235 & -0.2579 & 0.0757 & 0.0495 \\
\hline
\end{tabular}


Table 5. Comparison of $N u_{y} / R e_{y}^{1 / 2}$ values obtained in the present study with those obtained by Sparrow and Lin [26] for $G r_{y} / R e_{y}^{3}=0$ in power-law varying wall heat flux.

\begin{tabular}{ccccc}
\hline & & $\begin{array}{c}\text { Present } \\
\text { study }\end{array}$ & $\begin{array}{c}\text { Sparrow and } \\
\text { Lin [26] }\end{array}$ & Error (\%) \\
\hline \multirow{3}{*}{$\operatorname{Pr}=0.7$} & 0 & 0.45710 & 0.46320 & 0.013 \\
& 1 & 0.60550 & 0.60940 & 0.010 \\
$\operatorname{Pr}=10$ & 2 & 0.70470 & 0.70770 & 0.004 \\
& 0 & 0.45716 & 0.46318 & 0.004 \\
& 1 & 0.60544 & 0.60938 & 0.003 \\
& 2 & 0.70467 & 0.70766 & 0.005 \\
\hline
\end{tabular}

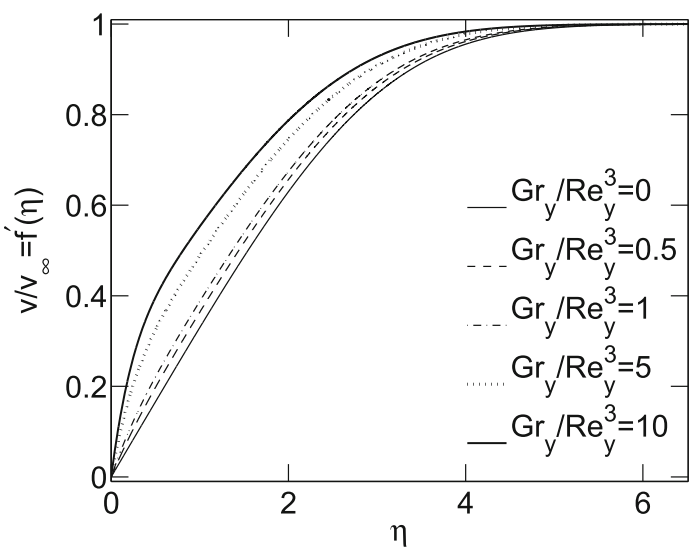

(a) $m=0$

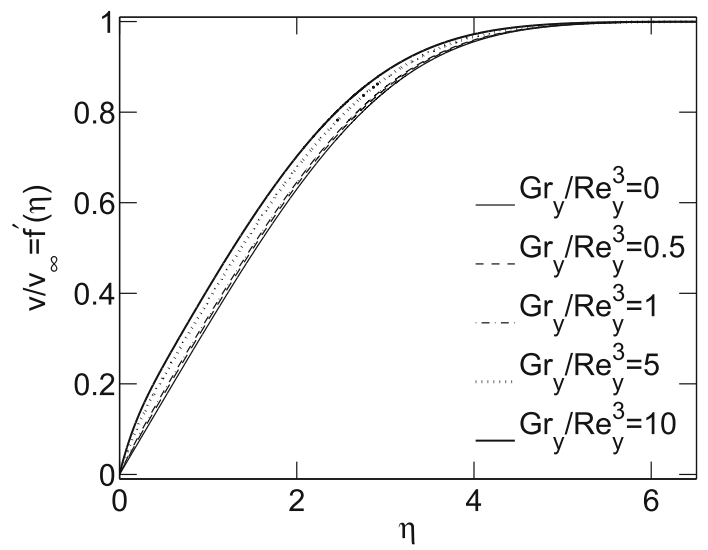

(b) $m=1$

Figure 5. Variation of dimensionless vertical velocity with $\eta$ for various values of $G r_{y} / R e_{y}^{3}$ in aiding flow.

variables. For a given wall flux variation, one can find out the values of perturbation variables of velocity and temperature to find out the skin friction drag and heat transfer rates along the wall. It may be noted that the calculations of skin friction drag and heat transfer rates for different boundary conditions involve only finding the perturbation variables, which require simple algebraic calculations, and

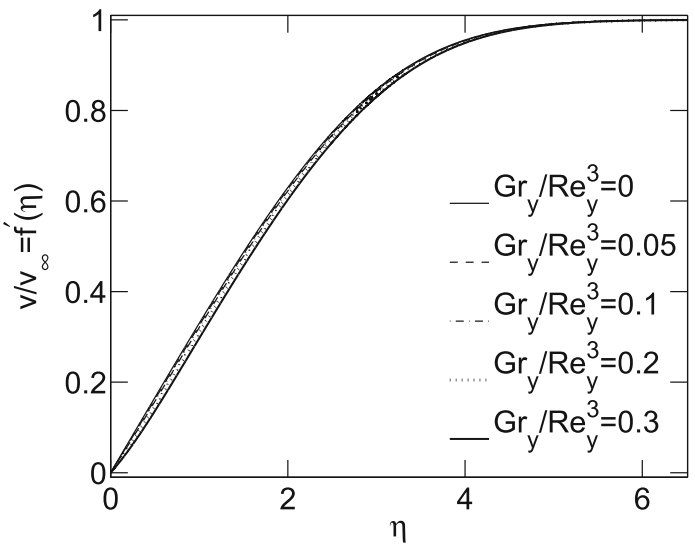

(a) $m=0$

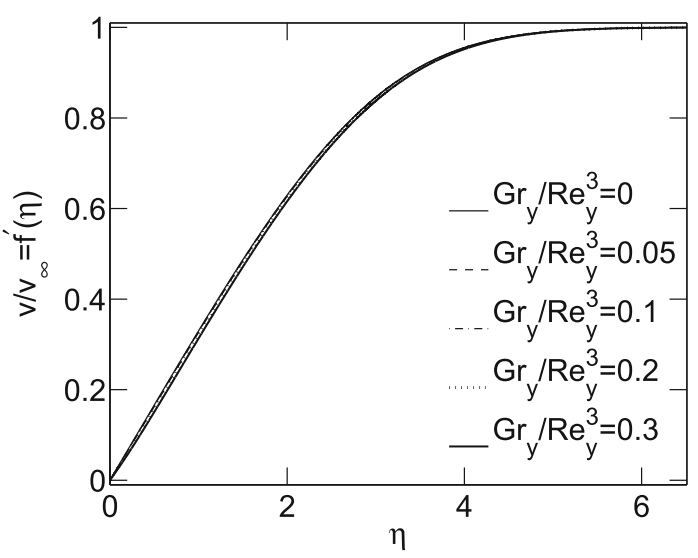

(b) $m=1$

Figure 6. Variation of dimensionless vertical velocity with $\eta$ for various values of $G r_{y} / R e_{y}^{3}$ in opposing flow.

we do not need to solve any differential equations. The universal functions of stream function (correspond to $\eta=0$ ) for aiding and opposing flows are shown in tables 1 and 2, which are required to calculate the skin friction drag. For calculation of heat transfer rates, the required universal functions of temperature (corresponding to $\eta=0$ ) are given in tables 3 and 4 for aiding and opposing cases, respectively, for various $G r_{y} / R e_{y}^{3}$ values.

\section{Mixed convection for power-law wall heat flux variation}

As these results are valid for any arbitrary wall heat flux variation, the results are applied for power-law wall flux variation. The results of power-law wall heat flux can be extended to any wall heat flux variation function that can be expanded in power-law series. The wall heat flux is assumed to vary in the form

$$
q_{w}(y)=A y^{m}
$$




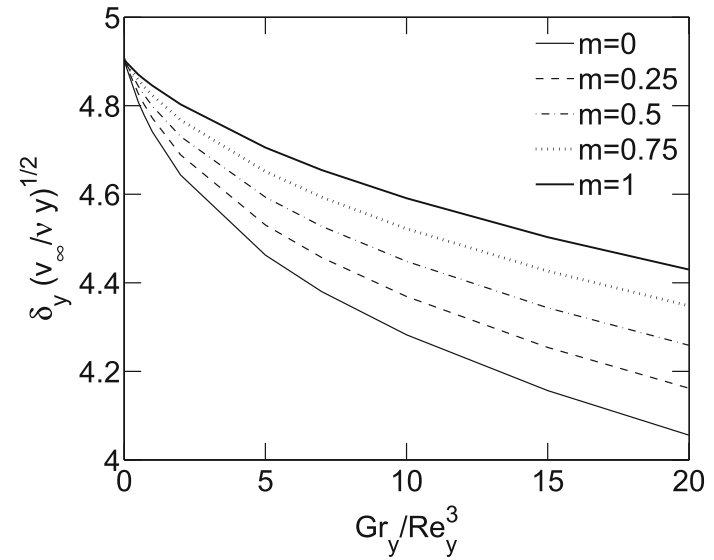

(a) Aiding

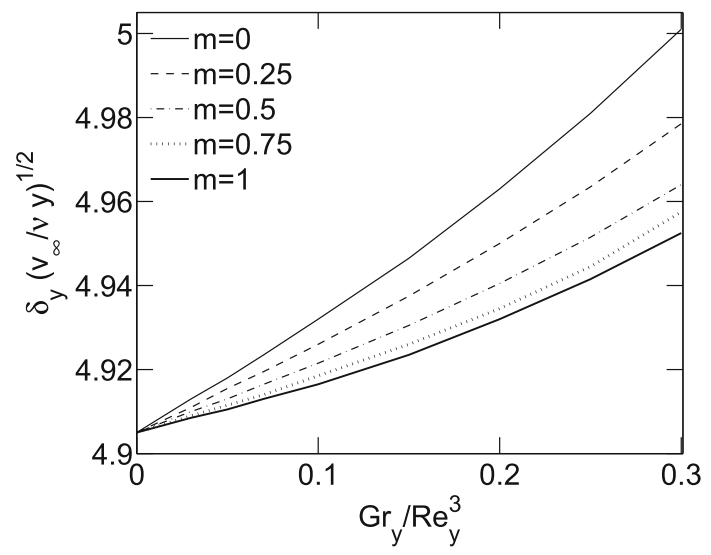

(b) Opposing

Figure 7. Variation of velocity boundary layer thickness with $G r_{y} / R e_{y}^{3}$ for various $m$ values.

where $A$ and $m$ are constants. The wall heat flux variation corresponds to constant heat flux when $m=0$.

For this form of wall heat flux variation, perturbation functions can be calculated using Eq. (21). The perturbation functions $\lambda_{0}, \lambda_{1}, \lambda_{0}^{2}, \ldots$ are as follows:

$$
\lambda_{0}=m, \quad \lambda_{1}=m(m-1), \quad \lambda_{0}^{2}=m^{2}, \quad \ldots
$$

For any given $m$ value, perturbation function values are calculated using Eq. (44). Once the perturbation function values are known, velocity, skin friction coefficient and Nusselt number calculations require the derivatives of universal functions with respect to $\eta$ in the expressions given by Eqs. (33), (36) and (42), respectively. These derivatives are available as part of the solutions of sets of ordinary differential equations; hence, they are known. Temperature is given by Eq. (23).

\subsection{Validation}

Before presenting results for the present problem, the code is validated by computing heat transfer rates for the special

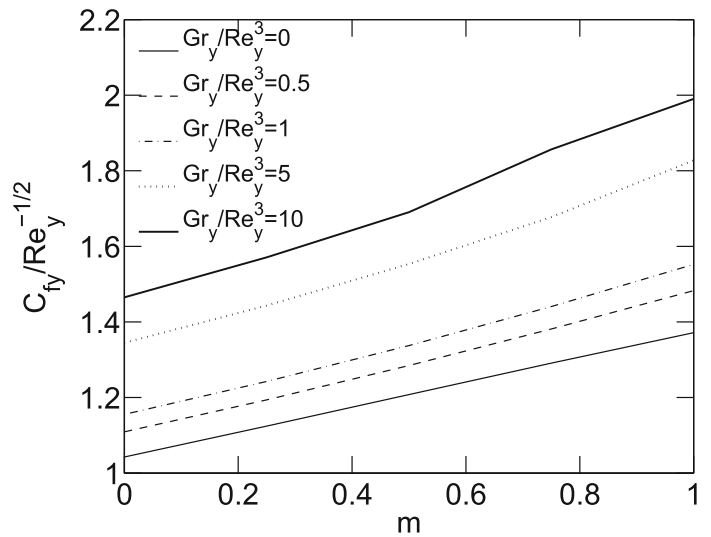

(a) aiding flow

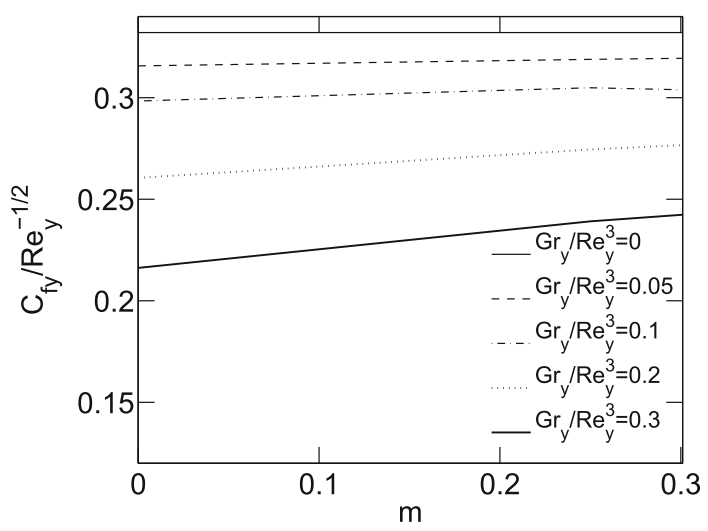

(b) opposing flow

Figure 8. Variation of skin friction coefficient with power index $m$ for various $G r_{y} / R e_{y}^{3}$.

case of forced convection for which results are available in Sparrow and Lin [26]. In the present study, $G r_{y} / R e_{y}^{3}=0$ corresponds to forced convection case. The heat transfer rates for forced convection obtained using Eqs. (44) and (42), by setting $G r_{y} / R e_{y}^{3}=0$ in the present study, are compared to those of Sparrow and Lin [26] in table 5. From table 5 we can see that the present results closely match with the reference results.

\subsection{Flow field calculations}

The dimensionless velocity variation is obtained using Eqs. (33) and (44) for various $G r_{y} / R e_{y}^{3}$ values and powerindex values of $m=0$ and $m=1$, which are presented in figures 5 and 6 . Figure 5 corresponds to aiding mixed convection, and figure 6 corresponds to opposing mixed convection. In these figures, the curves for $G r_{y} / R e_{y}^{3}=0$ correspond to forced convection extreme. It can be seen from the figures that velocities are greater than corresponding forced convection velocities in aiding mixed convection. However, in opposing mixed convection, the 


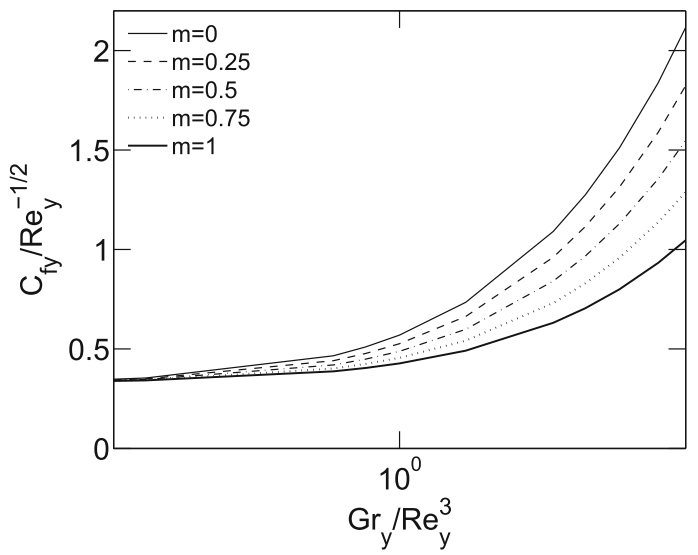

(a) aiding flow

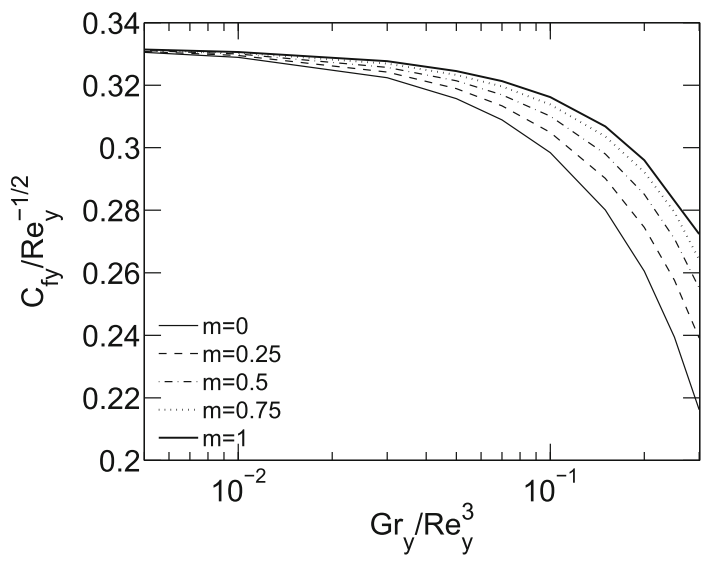

(b) opposing flow

Figure 9. Variation of skin friction coefficient with $G r_{y} / R e_{y}^{3}$ for various power index values $m$.

velocities are lower than corresponding forced convection velocities.

From the obtained velocity variations with $\eta$, we can find out the velocity boundary layer thickness $\delta_{v}\left(v_{\infty} / v y\right)^{1 / 2}$. The boundary layer thickness corresponds to $\eta$ at which $v / v_{\infty}$ becomes 0.99 . The variation of dimensionless boundary layer thickness with various $G r_{y} / R e_{y}^{3}$ for various $m$ values is shown in figure 7 , for both aiding and opposing situations. We can see from the figure that for a given $m$ value, $\delta_{v}\left(v_{\infty} / v y\right)^{1 / 2}$ decreases with increasing $G r_{y} / R e_{y}^{3}$ for aiding mixed convection. In this case, for a given $G r_{y} / R e_{y}^{3}, \delta_{v}\left(v_{\infty} / v y\right)^{1 / 2}$ increases with given $m$ values. The opposite trends can be observed for opposing mixed convection, in which $\delta_{v}\left(v_{\infty} / v y\right)^{1 / 2}$ increases with increasing values of $G r_{y} / R e_{y}^{3}$ and decreases with power index values $m$.

The variation of skin friction coefficient in the form of $C_{f y} / R e_{y}^{-1 / 2}$ with respect to $m$, for various $G r_{y} / R e_{y}^{3}$ values, is shown in figure $8 \mathrm{a}$ for aiding mixed convection. The figure corresponding to $G r_{y} / \operatorname{Re}_{y}^{3}=0$ is for forced convection

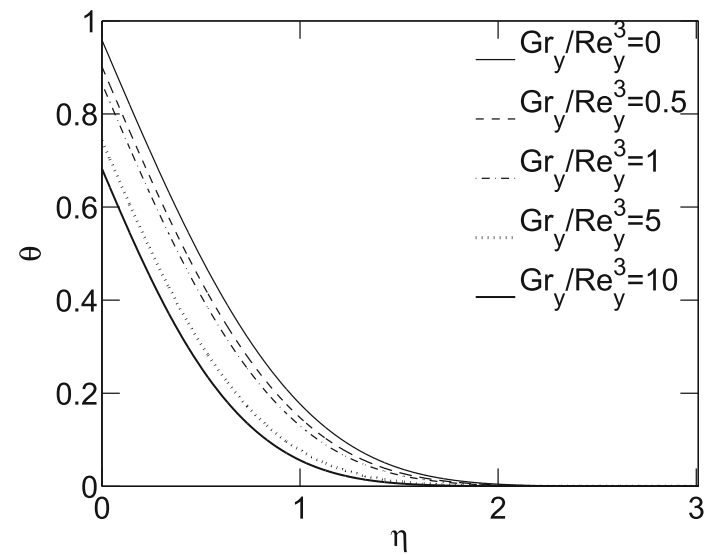

(a) $m=0$

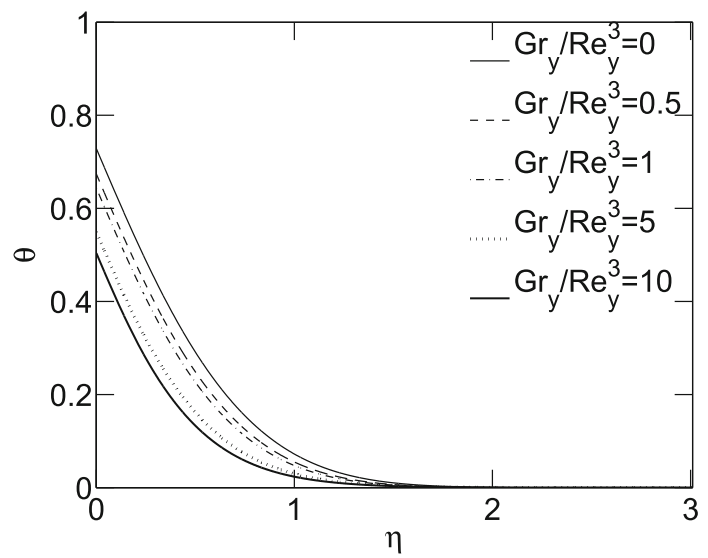

(b) $m=1$

Figure 10. Variation of dimensionless temperature with $\eta$ for aiding mixed convection.

and the skin friction coefficient increases with increasing $m$ values and also with values of $G r_{y} / R e_{y}^{3}$. The value of skin friction coefficient in mixed convection is higher than that of the forced convection and its value increases with $G r_{y} / R e_{y}^{3}$.

The variation of $C_{f y} / R e_{y}^{-1 / 2}$ with $m$ for various values of $G r_{y} / R e_{y}^{3}$ for opposing mixed convection is shown in figure $8 \mathrm{~b}$. The skin friction coefficient increases with increasing $m$ values. Skin friction coefficient values in opposing mixed convection are less than those of forced convection values. With increasing $G r_{y} / R e_{y}^{3}$ values, the value of $C_{f y} / R e_{y}^{-1 / 2}$ decreases, which is opposite to the trend of aiding mixed convection in which skin friction coefficient increases with increasing $G r_{y} / R e_{y}^{3}$. This is due to the fact that buoyancy and external forces cooperate with each other to increase the velocity in aiding flow, resulting in increasing skin friction coefficient. However, in the opposing case, these forces resist each other, resulting in decreased velocity, which shows decreased wall skin friction coefficient. In this opposing mixed convection, flow 


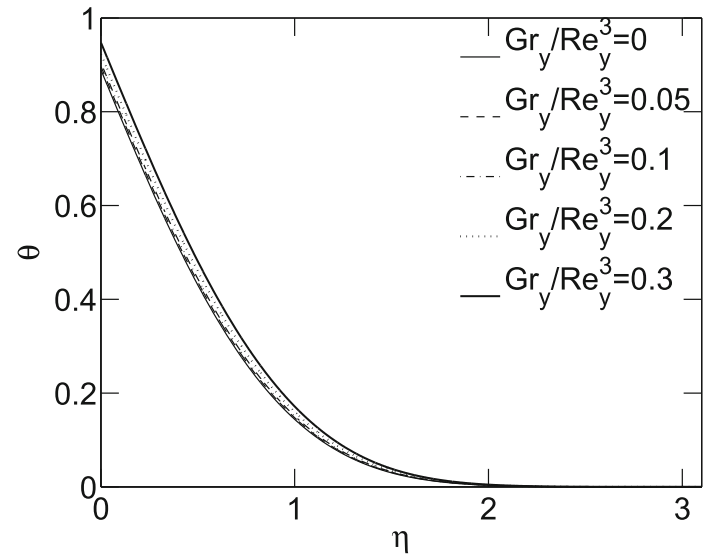

(a) $m=0$

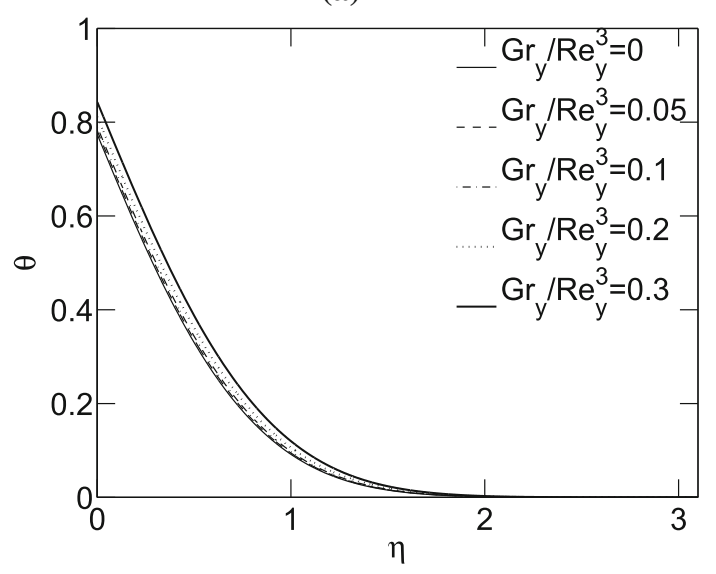

(b) $m=1$

Figure 11. Variation of dimensionless temperature with $\eta$ for opposing mixed convection.

reaches separation conditions for higher $G r_{y} / R e_{y}^{3}$ values. Hence, solutions are obtained for lower values of $G r_{y} / R e_{y}^{3}$ in opposing mixed convection.

The variation of $C_{f y} / R e_{y}^{-1 / 2}$ with respect to $G r_{y} / R e_{y}^{3}$ for various power index values is shown in figure $9 \mathrm{a}$ and $\mathrm{b}$, respectively, for aiding and opposing mixed convection. At $G r_{y} / R e_{y}^{3}=0$, the plot shows the skin friction coefficient for forced convection flow. In aiding mixed convection, skin friction coefficient values start from forced convection values and increase towards natural convection asymptote, with increasing $G r_{y} / R e_{y}^{3}$ values. In the case of opposing mixed convection flow, skin friction coefficient begins with forced convection values and decreases as $G r_{y} / R e_{y}^{3}$ increases. When $G r_{y} / R e_{y}^{3}$ is increased till the skin friction coefficient becomes zero, flow separation occurs, beyond which the boundary layer approximations are not applicable.

\subsection{Heat transfer calculations}

The variation of $\theta$ is presented for various $G r_{y} / R e_{y}^{3}$ values for $m=0$ and $m=1$, and shown in figures 10 and 11 for

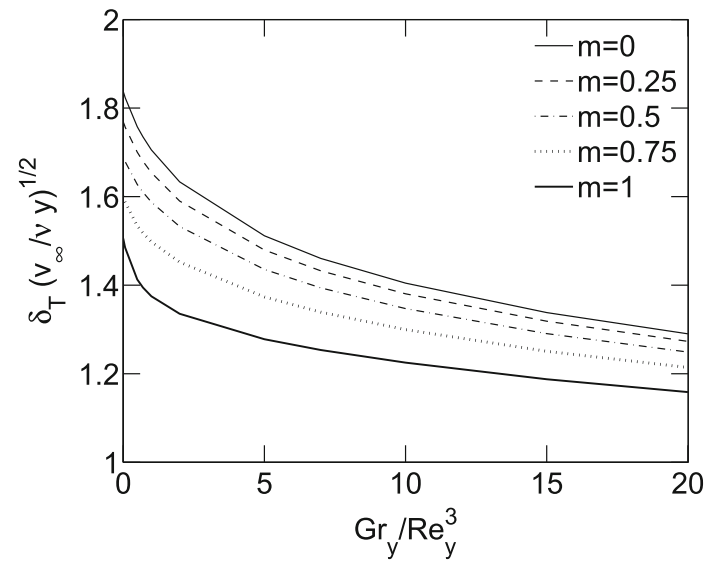

(a) Aiding

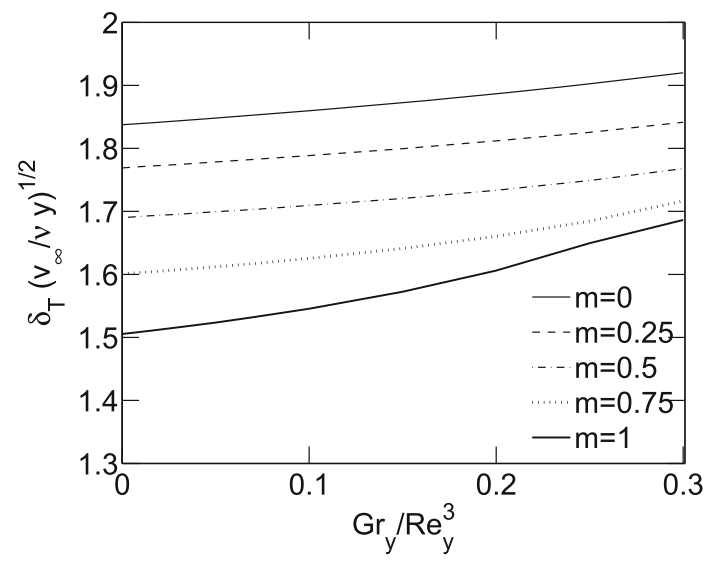

(b) Opposing

Figure 12. Variation of thermal boundary layer thickness with $G r_{y} / R e_{y}^{3}$ for various $m$ values in mixed convection.

aiding and opposing mixed convection, respectively. From the figures, we can see that for a given $\eta$ value, values of $\theta$ decrease with increasing values of $G r_{y} / R e_{y}^{3}$ in case of aiding mixed convection. However, the opposite trend is found for opposing mixed convection.

From the $\theta$ variation with $\eta$, the thermal boundary layer thickness $\delta_{T}\left(v_{\infty} / v y\right)^{1 / 2}$ can be found. From this $\eta$ value, boundary layer thickness can be calculated using Eq. (14). The thermal boundary layer thickness corresponds to $\eta$ value at which dimensionless temperature $\theta$ equals 0.01 . The thermal boundary layer thickness is found for various $G r_{y} / R e_{y}^{3}$ and $m$ values, which are derived in dimensionless form as $\delta_{T}\left(v_{\infty} / v y\right)^{1 / 2}$. The variation of boundary layer thickness with $G r_{y} / R e_{y}^{3}$ for various $m$ values is presented in figure 12 for both aiding and opposing mixed convection. The figure shows that the boundary layer thickness at a given $y$ location decreases with increasing $G r_{y} / R e_{y}^{3}$ values in aiding mixed convection. However, for opposing mixed convection, boundary layer thickness increases with $G r_{y} / R e_{y}^{3}$ values. 


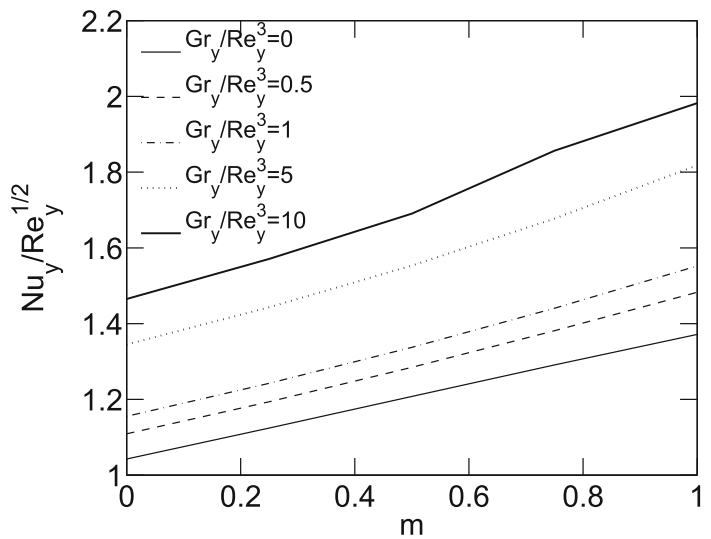

(a) aiding flow

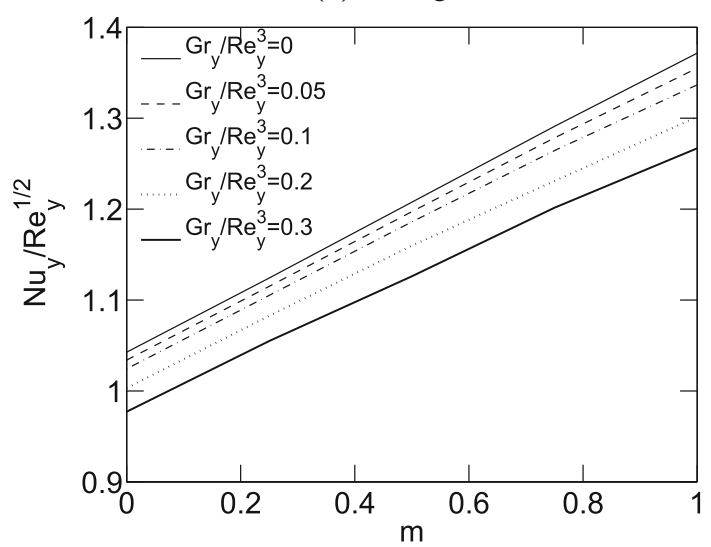

(b) opposing flow

Figure 13. Variation of heat transfer rate with $m$ for various $G r_{y} / R e_{y}^{3}$ values in aiding and opposing flows.

The heat transfer rates calculated using Eqs. (42) and (44) are presented in tables 3 and 4 . The heat transfer rates are calculated in dimensionless form of $N u_{y} / R e_{y}^{1 / 2}$ for various values of $m$ and $G r_{y} / R e_{y}^{3}$. The values for $m=0$ correspond to the case of uniform wall heat flux.

The variation of $N u_{y} / R e_{y}^{1 / 2}$ with power index value $m$ for various values of $G r_{y} / R e_{y}^{3}$ is shown in figure 13a and b, respectively, for aiding and opposing mixed convection. The Nusselt number increases with power index for all $G r_{y} / R e_{y}^{3}$ values. The plot for $G r_{y} / R e_{y}^{3}=0$ in the figures corresponds to forced convection asymptote. The heat transfer rates increase with increasing $G r_{y} / R e_{y}^{3}$ and wall heat flux power-index value. The value of $N u_{y} / R e_{y}^{1 / 2}$ varies almost linearly with power index $m$ in the range of $0-1$. In aiding mixed convection, heat transfer rates are higher than those of forced convection values. Heat transfer rates increase with increasing values of $G r_{y} / R e_{y}^{3}$.

In opposing mixed convection, heat transfer rates are less than those corresponding to forced convection. The heat transfer rates decrease with increasing $G r_{y} / R e_{y}^{3}$. This is opposite to the trend of aiding mixed convection. This is due to the fact that in aiding mixed convection, buoyancy forces act in the same direction of external forces and result in increasing flow velocities. The increased flow velocities result in high heat transfer rates. In opposing mixed convection, buoyancy forces oppose external forces, resulting in separation of convection. In this case, heat transfer rates are lower than that of forced convection values. It may be noted that the opposing mixed convection is solved for $G r_{y} / R e_{y}^{3}$ values lower than those values for which aiding mixed convection is solved. This is because for higher $G r_{y} / R e_{y}^{3}$ values in the opposing mixed convection, boundary layer tends towards flow separation, where boundary layer approximations are no longer applicable. Hence, opposing mixed convection is solved for lower values of $G r_{y} / R e_{y}^{3}$.

To study the effect of $G r_{y} / R e_{y}^{3}$ on heat transfer rates, the variation of $N u_{y} / R e_{y}^{1 / 2}$ with $G r_{y} / R e_{y}^{3}$ is plotted for various values of $m$ in figure $14 \mathrm{a}$ and $\mathrm{b}$ for aiding and opposing mixed convection, respectively. In the figures, the plot for $G r_{y} / R e_{y}^{3}=0$ corresponds to forced convection asymptote. From the figures, we can see that heat transfer rates start

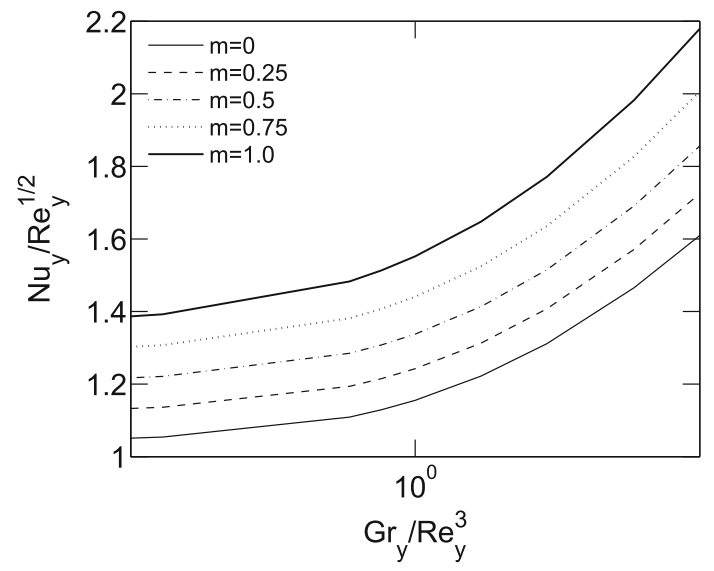

(a) aiding flow

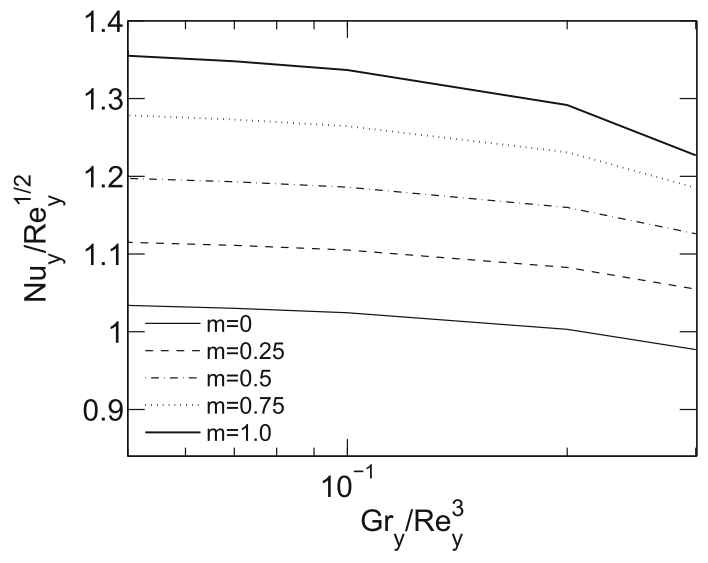

(b) opposing flow

Figure 14. Variation of heat transfer rate with $G r_{y} / R e_{y}^{3}$ at various $m$ values for aiding and opposing flows. 
with forced convection values and vary towards natural convection with increasing values of $G r_{y} / R e_{y}^{3}$. This is typical in mixed convection heat transfer.

\section{Conclusions}

The steady laminar boundary layer mixed convection of water over vertical flat surface of varying heat flux with density inversion is solved using perturbation technique. Heat flux differentials are used as perturbation elements. The solutions are obtained in terms of universal functions that are valid for any arbitrary wall heat flux variation. The solutions are applied to power-law varying wall heat flux condition. The velocity and temperature distributions in the boundary layer, boundary layer thickness, local skin friction coefficient and heat transfer rates along the wall are presented for various $G r_{y} / R e_{y}^{3}$ values and power-law index values. It is observed that, in the parameter range of the present study, heat transfer rates and skin friction coefficient are found to increase almost linearly with wall flux power index, for a given combination of Grashof and Reynolds numbers, for both the cases of aiding and opposing mixed convection. The solutions from perturbation method have the advantage that the solutions are valid for any arbitrary wall heat flux variation. Solutions for different wall heat flux varying conditions can be obtained using simple algebraic calculations without the need for solving any differential equations.

\section{Nomenclature}

$A$

$C_{f}$

$f$

$F, f_{0}, f_{1}$,

$\ldots, f_{00}, f_{11}(\eta), \ldots$

$g$

$G r$

$h$

$H, \theta_{0}, \theta_{1}$, $\ldots, \theta_{00}, \theta_{11}(\eta), \ldots$ $k$

$m$

$\mathrm{Nu}$
$\mathrm{Pr}$
$q$
$\operatorname{Re}$
$T$
$T_{0}$

constant in power-law wall heat flux variation (Eq. (43))

skin friction coefficient non-dimensional stream function universal functions for velocity in perturbation technique gravitational acceleration $\left(\mathrm{m} / \mathrm{s}^{2}\right)$ modified Grashof number heat transfer coefficient $\left(\mathrm{W} / \mathrm{m}^{2} \mathrm{~K}\right)$ universal functions for temperature in perturbation technique thermal conductivity of fluid (W/m K) power index of power-law variation of wall heat flux

Nusselt number Prandtl number heat flux $\left(\mathrm{W} / \mathrm{m}^{2}\right)$ Reynolds number temperature $(\mathrm{K})$ temperature corresponding to maximum density of water near density inversion $(\mathrm{K})$

$\begin{array}{ll}u, v & \text { velocity components in } x \text { and } y \\ & \text { directions, respectively }(\mathrm{m} / \mathrm{s}) \\ v_{\infty} & \text { free stream velocity }(\mathrm{m} / \mathrm{s}) \\ x, y & \text { Cartesian coordinates }(\mathrm{m})\end{array}$

\section{Greek symbols}

$\alpha \quad$ thermal diffusivity $\left(\mathrm{m}^{2} / \mathrm{s}\right)$

$\delta_{v} \quad$ velocity boundary layer thickness (m)

$\delta_{T} \quad$ thermal boundary layer thickness (m)

$\eta \quad$ non-dimensional independent variable

$\gamma \quad$ constant in Eq. (1) on density

variation of water with temperature

$\lambda_{0}, \lambda_{1}, \quad$ a set of variables that

$\ldots, \lambda_{n}$ are functions of $y$

$v \quad$ kinematic viscosity $\left(\mathrm{m}^{2} / \mathrm{s}\right)$

$\psi \quad$ dimensional stream function

$\rho \quad$ density $(1 /$ specific volume $)\left(\mathrm{kg} / \mathrm{m}^{3}\right)$

$\rho_{0} \quad$ maximum density of water $\left(\mathrm{kg} / \mathrm{m}^{3}\right)$

$\theta \quad$ non-dimensional temperature

\section{Subscripts}
w wall
y local quantity
$\infty$ free stream quantity

\section{References}

[1] Wilks G 1974 The flow of a uniform stream over a semiinfinite vertical flat plate with uniform surface heat flux. Int. J. Heat Mass Transfer 17: 743-753

[2] Carey V P and Gebhart B 1982 Transport at large downstream distances in mixed convection flow adjacent to a vertical uniform heat flux surface. Int. J. Heat Mass Transfer 25(2): 255-266

[3] Moulic S G and Yao L S 2009 Mixed convection along a semi-infinite vertical flat plate with uniform surface heat flux. J. Heat Transfer-Trans. ASME 131: 225021-225028

[4] Merkin J H and Mahmood T 1989 Mixed convection boundary layer similarity solutions: prescribed wall heat flux. J. Appl. Math. Phys. 40: 51-68

[5] Merkin J H, Pop I and Mahmood T 1991 Mixed convection on a vertical surface with a prescribed heat flux: the solution for small and large Prandtl numbers. J. Eng. Math. 25: 165-190

[6] Wickern G 1991 Mixed convection from an arbitrarily inclined semi-infinite flat plate-I, the influence of the inclination angle. Int. J. Heat Mass Transfer 34(8): 1935-1945

[7] Wickern G 1991 Mixed convection from an arbitrarily inclined semi-infinite flat plate-II. The influence of the Prandtl number. Int. J. Heat Mass Transfer 34(8): 1947-1957

[8] Yeh H M, Tsai S W and Yang C C 1987 Heat and mass transfer in mixed convection over a horizontal plane. Numer. Heat Transfer 12(2): 229-242

[9] Armaly B F, Chen T S and Ramachandran N 1987 Correlations for laminar mixed convection on vertical, inclined 
and horizontal flat plates with uniform surface heat flux. Int. J. Heat Mass Transfer 30(2): 405-405

[10] Trimbitas R, Grosan T and Pop I 2015 Mixed convection boundary layer flow past vertical flat plate in nano fluid: case of prescribed wall heat flux. Appl. Math. Mech. 36(8): 1091-1104

[11] Ranganathan P and Viskanta R 1984 Mixed convection boundary-layer flow along a vertical surface in a porous medium. Numer. Heat Transfer 7(3): 305-317

[12] Ahmad S and Pop I 2010 Mixed convection boundary layer flow from a vertical flat plate embedded in a porous medium filled with nano fluids. Int. Commun. Heat Mass Transfer 37: 987-991

[13] Mahmoud M A A 2010 Variable fluid properties and thermal radiation effects on mixed convection flow over a horizontal surface. Int. J. Comput. Methods Eng. Sci. Mech. 11: 299-303

[14] Sawant S M and Rao C G 2010 Combined conduction-mixed convection-surface radiation from a uniformly heated vertical plate. Chem. Eng. Commun. 197: 881-899

[15] Pal D 2015 Unsteady convective boundary layer flow and heat transfer over a stretching surface with non-uniform heat source/sink and thermal radiation. Int. J. Comput. Methods Eng. Sci. Mech. 16(3): 170-181

[16] Vighnesam N V and Soundalgekar V M 1997 Combined free and forced convection flow of water at $4{ }^{\circ} \mathrm{C}$ from a vertical plate with variable temperature. Indian J. Eng. Mater. Sci. 5: 124-126

[17] Lin D S and Gebhart B 1986 Buoyancy-induced flow adjacent to a horizontal surface submerged in porous medium saturated with cold water. Int. J. Heat Mass Transfer 29(4): 611-623

[18] Kumaran V and Pop I 2006 Steady free convection boundary layer over a vertical flat plate embedded in a porous medium filled with water at $4^{\circ}$ C. Int. J. Heat Mass Transfer 49: 3240-3252

[19] Hussain T and Afzal N 1988 Mixed convection boundary layer flow on a horizontal plate in a uniform stream. Int. J. Heat Mass Transfer 31(12): 2505-2516

[20] Kumari M 2001 Variable viscosity effects on free and mixed convection boundary-layer flow from a horizontal surface in a saturated porous medium - variable heat flux. Mech. Res. Commun. 28(3): 339-348

[21] Gavara M R, Dutta P and Seetharamu K N 2012 Mixed convection adjacent to non-isothermal vertical surfaces. Int. J. Heat Mass Transfer 55(17-18): 4580-4587

[22] Seetharamu K N and Dutta P 1988 Mixed convection about a non-isothermal vertical surface in a porous medium. Int. J. Numer. Methods Fluids 8(6): 723-735

[23] Tong W and Koster J N 1993 Natural convection of water in a rectangular cavity including density inversion. Int. J. Heat Fluid Flow 14(4): 366-375

[24] Schlichting H and Gersten K 2001 Boundary layer theory, 8th ed. Berlin, Heidelberg: Springer-Verlag

[25] Van Dyke M D 1975 Perturbation methods in fluid mechanics. New York: Parabolic Press

[26] Sparrow E M and Lin S H 1965 Boundary layers with prescribed heat flux-application to simultaneous convection and radiation. Int. J. Heat Mass Transfer 8(3): 437-448 